\title{
1 Analysis methods for measuring fNIRS 2 responses generated by a block-design 3 paradigm
}

4 Robert Luke ${ }^{1,3}$, Eric Larson 7 , Maureen J Shader ${ }^{3,5}$, Hamish Innes-Brown ${ }^{4,5}$, Lindsey Van Yper ${ }^{1}$ 5 Adrian KC Lee ${ }^{2,7}$, Paul F Sowman ${ }^{6}$, David McAlpine ${ }^{1}$

6 1. Macquarie University Hearing \& Department of Linguistics, Australian Hearing Hub, Macquarie University, 7 Sydney, Australia

8 2. Department of Speech \& Hearing Sciences and Institute for Learning \& Brain Sciences, University of 9 Washington, Seattle, WA, USA

10 3. The Bionics Institute Melbourne

11 4. Eriksholm Research Centre, Oticon A/S

12 5. Department of Medical Bionics, The University of Melbourne

13 6. Department of Cognitive Science, Faculty of Medicine, Health and Human Sciences, Macquarie University, 14 Sydney, Australia

15 7. Institute for Learning \& Brain Sciences, University of Washington, Seattle, WA, USA

17 Overview

Significance: fNIRS is an increasingly popular tool in auditory research, but the range of analysis procedures employed across studies complicates interpretation of data.

Aim: To assess the impact of different analysis procedures on the morphology, detection, and lateralization of auditory responses in fNIRS. Specifically, whether averaging or GLM-based analyses generate different experimental conclusions, when applied to a block-protocol design. The impact of parameter selection of GLMs on detecting auditory-evoked responses was also quantified.

Approach: 17 listeners were exposed to three commonly employed auditory stimuli: noise, speech, and silence. A block design was employed, comprising sounds of 5-s duration, and 10-20 s silent intervals. 
Results: Both analysis procedures generated similar response morphologies and amplitude estimates, and both also indicated responses to speech to be significantly greater than to noise and silence. Neither approach indicated a significant effect of brain hemisphere on responses to speech. Methods to correct for systemic hemodynamic responses using short channels improved detection at the individual level.

Conclusions: Consistent with theoretical considerations, simulations, and other experimental domains, GLM and averaging analyses generate the same group-level experimental conclusions. We release this dataset publicly for use in future development and optimization of algorithms.

\section{Introduction}

Functional near-infrared spectroscopy (fNIRS) is an increasingly popular technique (Yücel et al., 2017) employed to investigate auditory-cortical function, and provides for a unique set of qualities that make it ideal for auditory research. fNIRS devices are typically very quiet compared to functional magnetic resonance imaging (fMRI) with which it shares a similar biologically generated signal. fNIRS is unaffected by electrical or magnetic interference from

44 hearing devices such as cochlear implants or hearing aids, all of which are either contraindicated or generate large artifacts in fMRI as well as in electro- and magnetoencephalography (EEG and MEG, respectively). fNIRS devices are generally relatively portable

47 and do not require participants or patients to be isolated in a shielded chamber, or to have their head-position fixed, making it well suited for use in low- or non-compliant groups, 
49 including children, the elderly, and the cognitively impaired. It therefore provides an ideal

50 imaging modality for clinical applications.

51 fNIRS has been used to investigate a variety of auditory research questions and applications.

52 A primary use has been the investigation of cortical processing of physical qualities of sound,

53 such as intensity, and amplitude and frequency modulations, and auditory-spatial cues

54 (Weder et al., 2020; Weder et al., 2018; Zhang et al., 2018). fNIRS has also been employed to evaluate the perceptual qualities of speech and listening effort, as well as language development in normal-hearing and hearing-impaired populations (Anderson et al., 2019; Lawrence et al., 2018; Mushtaq et al., 2019; Pollonini et al., 2014; Rovetti et al., 2019; Rowland et al., 2018; Sevy et al., 2010; Wiggins et al., 2016b; Wijayasiri et al., 2017; Zhang et al., 2020). Research questions relating to the development of auditory cortical function

60 (Gervain et al., 2008), and cortical reorganization following impaired sensory input and subsequent rehabilitation (Anderson et al., 2017; Wiggins and Hartley, 2015) have been investigated using fNIRS, as have outcomes related to cochlear implantation (Anderson et al., 2019) and auditory pathologies such as tinnitus (Basura et al., 2018; Shoushtarian et al., 2020).

Despite this utility, however, relative to other neuroimaging modalities such as fMRI, EEG, and MEG, fNIRS has been employed only recently by hearing scientists, and considerable variability exists in the experimental designs and analysis techniques used by different researchers. This variability can make it difficult to interpret data sets, or to replicate or compare findings across studies, or between research teams. The experimental designs most commonly employed by auditory fNIRS researchers are block- and event-related designs. 
72 statistical power of the protocol, the duration of the experiment, and whether the design provides the flexibility to study the effect of interest ( Birn et al., 2002; Friston et al., 1999; Henson, 2007; Mechelli et al., 2003). For example, an event-related design may enable an investigator to examine the response to individual words in an ongoing sentence, something not possible when employing a block design.

Here, we compare two common analysis procedures that can be applied in experiments employing a block design. Block-design experiments present a single stimulus type continuously for an extended time interval (e.g. 5 s), followed by an inter-stimulus interval (i.e., where no stimulus is presented) of sufficient duration for the hemodynamic response to return to an approximate basal level (Brockway, 2000; Rombouts et al., 1997). Although commonly employed, no consensus exists as to the most appropriate analysis procedures for this type of experimental design; new algorithms and procedures are regularly published without cross-validation or theoretical consideration.

Analysis procedures for block designs typically lie in one of two categories: averaging analysis, where the fNIRS measurement is segmented and averaged relative to the onset of the stimulus (Dawson, 1954); and general linear model (GLM) analysis, where one or more model hemodynamic responses are fitted to the entirety of the measured fNIRS signal (Cohen, 1997; and for a recent overview in the context of fNIRS see Huppert, 2016). The signal averaging approach assumes that the noise component of the measured fNIRS signal is a random process with zero mean, and unrelated to the biological signal of interest. In contrast, the

92 GLM is capable of accounting for a more complex model of signal noise (Barker et al., 2013). Although for non-overlapping responses such as are assumed in a block design, the GLM model is reduced to a block average, suggesting that both analyses should tend to generate 
similar outcomes (Dale and Buckner, 1997; Santosa et al., 2019), due to the statistical properties of the fNIRS signal, GLM analysis may be a more appropriate method with which to analyze fNIRS data (Huppert, 2016). These two analysis methods have been described and evaluated for different fNIRS analysis parameters in computer simulations and behavioral motor experiments (Santosa et al., 2019; Tak and Ye, 2014), but a direct comparison has yet to be made for research investigating audition.

In general, auditory-cortical responses in fNIRS have been shown to be reliable at a group level (Wiggins et al., 2016a). Many investigations of auditory cortical function target relatively deep (relative to the skull) cortical regions such as Heschl's gyrus, of which a typical fNIRS device might generate less than $1 \%$ specificity (Zimeo Morais et al., 2018). This low specificity makes individual-level measurements unreliable, largely due to the poor signal-to-noise ratio; the measured stimulus-evoked hemodynamic response is small compared to all other sources of bio-generated changes in the fNIRS signal. This challenge has motivated the need for a comparison of averaging and GLM analysis specifically for auditory fNIRS signals, in order to understand the influence of analysis choices when analyzing such a small signal-of-interest. Here, we investigate whether averaging and GLM style analysis applied to the same dataset generate data that support the same experimental conclusions.

Due to the statistical properties of the noise within fNIRS signals, GLM-style analysis has been suggested to be a more appropriate method with which to analyze fNIRS data (Huppert, 2016). As such, we also investigated the influence of the parameters employed in GLM analysis on the true and false detection-rates of sound-generated fNIRS responses. Of particular importance in fNIRS experiments is the separation (and possible reduction) of systemic contributions (changes in the measured fNIRS signal that are not due to the effect 
of neurovascular coupling) to the measured signal when estimating neural responses

119 (Tachtsidis and Scholkmann, 2016). This has particular relevance for auditory experiments, as systemic components of fNIRS measurements have been shown to be related to the

121 characteristics of acoustic stimuli (Shoushtarian et al., 2019).

122 Many approaches have been proposed to remove the influence of systemic components on 123 the estimation of the neural response (Fabbri et al., 2004; Saager and Berger, 2005; Santosa 124 et al., 2020; Scholkmann et al., 2014; Wyser et al., 2020). Most use specialized channels designed not to measure neural activity but the systemic response only. These channels typically have a source-detector separation of less than $1 \mathrm{~cm}$, and are often referred to as 'short' channels. Recently, Santosa et al. (2020) concluded that including short-channel information as a regressor of no interest within a GLM analysis resulted in the most accurate estimation of the underlying neural response compared to spatial and temporal filtering, regression, and component analysis.

131 We therefore investigated the effect of including information from short channels on the detection of auditory fNIRS responses. Algorithms that remove systemic components have previously been evaluated and contrasted (Santosa et al., 2020; Scholkmann et al., 2014; Wyser et al., 2020), but we apply these methods specifically in the context of two commonly used auditory stimuli: speech and band-pass noise.

136 Speech is the primary mode for auditory communication, and is therefore widely employed 137 in auditory experiments. Noise signals are often used to investigate basic auditory processing, 138 as the statistical properties of the signal can be precisely controlled. These two stimuli are often contrasted to investigate language-specific processing, or combined to investigate speech processing in challenging listening environments. Both stimuli can hold an infinite 
number of forms; speech may contain prosodic cues or be spectrally degraded, and noise may

142 comprise different frequency ranges, contain modulations in amplitude or frequency, or

143 transition over time. Here, we employed two different stimuli: speech comprising three

144 concatenated sentences in quiet, and a 400- $\mathrm{Hz}$ band of noise centered at $500 \mathrm{~Hz}$.

145 We first describe the methods used to produce and present stimuli, and to generate data. We

146 then undertake qualitative analysis examining the morphology of fNIRS responses to auditory

147 stimuli using averaging and GLM analyses, and assess the influence of different analysis parameters on the detection of auditory fNIRS responses, and on the rate of false positives.

149 Finally, we investigate whether the averaging and GLM approaches provide similar experimental conclusions when applied to the same dataset. Both approaches were used to investigate two common questions in auditory neuroscience. First, do two different stimulus conditions generate a different response amplitude? Second, are cortical-hemispheric difference apparent in evoked responses?

154 One challenge when developing an experimental protocol for fNIRS is to understand the effects of different analysis choices, and to optimize the signal-processing procedure. Further,

156 it is important not to optimize a specific analysis pipeline using the same data from which 157 scientific conclusions will be drawn (Kriegeskorte et al., 2009). The dataset we report here will be released publicly to assist in the development of future auditory fNIRS pipelines and algorithm development. In a similar vein, we note that that we are not endeavouring to generate scientific conclusions concerning the relative cortical processing of speech and noise. Rather, our intention is to provide an understanding of the choice of parameters on conclusions reached by statistical analysis of auditory-generated fNIRS responses generated using averaging and GLM techniques. 


\section{Methods}

\subsection{Experimental Design}

167 Seventeen participants volunteered for this project. All participants indicated no history of hearing concerns. Participants were aged between 22 and 40 years. Data were collected under the Macquarie University Ethics Application Reference 52020640814625.

Participants were seated in a sound-attenuating booth in a comfortable chair for the duration

171 of the experiment, which lasted approximately 25 minutes. Participants were instructed not to pay attention to the sounds and were offered the choice of watching a silent, subtitled film during the experiment; seven participants accepted this option. NIRS data were recorded using a NIRx NIRScoutX device with APD detectors. The data were saved to disk with a sample rate of $5.2 \mathrm{~Hz} .12$ source channels and 12 detector channels were employed in the fNIRS optode-cap configuration, with eight additional short detectors distributed across the head.

177 Sources were placed at the positions AF7, F3, F7, FC5, T7, CP5, O1, POz, O2, Iz, CP6, and T8.

178 Detectors were placed at the positions F5, C5, TP7, CP3, P5, PO3, P04, Oz, P6, CP4, TP8, and 179 C6. Short detectors were placed at AF7, F7, T7, CP5, 01, O2, CP6, and T8 (Figure 1). These 180 optodes were selected to target four regions of interest (ROI) using the fOLD toolbox (Zimeo 181 Morais et al., 2018), including the left inferior frontal gyrus (IFG), the left and right superior 182 temporal gyri (STG), and the occipital lobe. The left inferior frontal gyrus is indicated in speech and language processing, whilst the superior temporal gyri are indicated in auditory processing. The occipital lobe is indicated in visual processing and as a possible additional site 
(a)

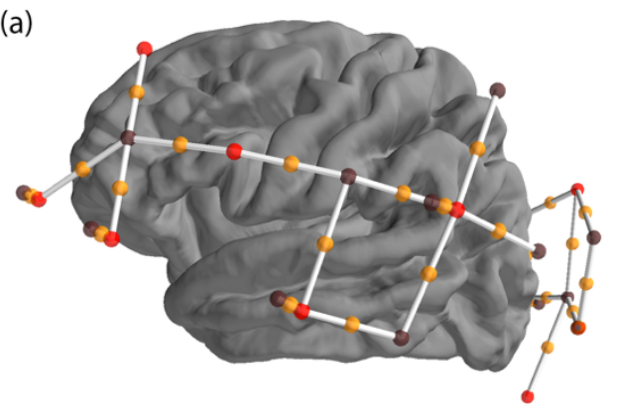

(b)

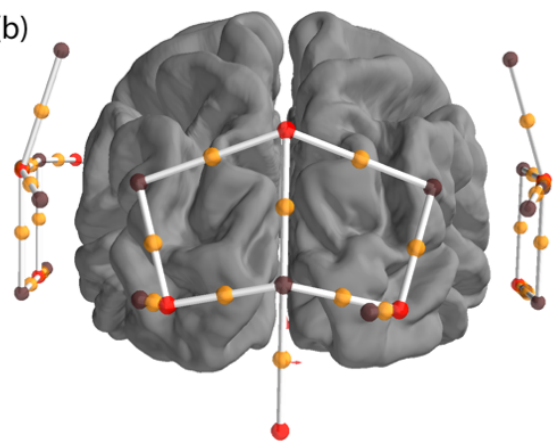

(c)

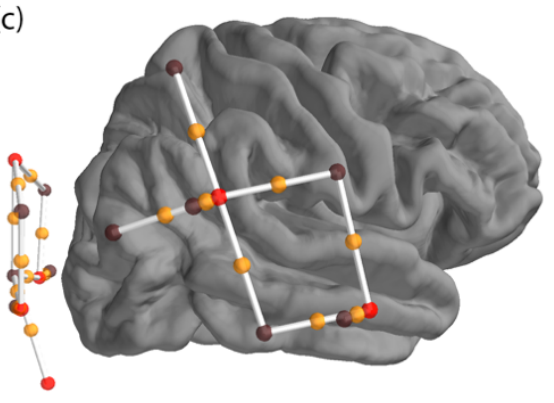

189

190

191

192

193

194

195

196

197

198

199

200

201

202

203

Figure 1: Location of sources and detectors. Four regions of interest were created to cover the left inferior frontal gyrus, the left and right superior temporal gyri, and the occipital region. Sources are shown as red dots, detectors are shown as black dots, channels are shown as white lines with an orange dot representing the midpoint. The montage is shown from the left (a), back (b) and right (c) views of the brain.

Participants listened to auditory stimuli presented diotically (i.e., the same sound to both ears) via Etymotic Research ER-2 insert-phones connected to an RME Fireface UCX soundcard (16 bits, $44.1 \mathrm{kHz}$ sampling rate). Speech was presented at $80 \mathrm{~dB}$ SPL, and noise (separately) at $85 \mathrm{~dB}$ SPL. Stimuli were calibrated to a Casella Cel-110/2 sound source using a Norsonic sound-level meter (Norsonic SA, Norway) and an ear simulator (RA0045 G.R.A.S., Denmark).

Participants were exposed to three stimulus conditions: speech, noise, and silence. The speech stimulus consisted of three concatenated sentences from the AusTIN speech corpus (Dawson et al., 2013) with a total duration of $5.25 \mathrm{~s}$. The noise stimulus consisted of a uniform distribution of frequency content between $300-700 \mathrm{~Hz}$, and was of 5-s duration. Five seconds of silence was used as the control condition. Stimuli were presented in random order with an inter-stimulus interval selected randomly for each trial from a uniform distribution in the range $10-20 \mathrm{~s}$. Twenty trials were presented for each condition, resulting in a total of 60 trials per participant. 


\subsection{Analysis}

208 All analyses were performed using MNE (version 0.21.2) (Gramfort et al., 2013; Gramfort et

209 al., 2014) and MNE-NIRS (version 0.0.1) (https://mne.tools/mne-nirs/), which makes extensive use of the Nilearn package (version 0.70) (Abraham et al., 2014) for GLM analysis. First, a qualitative analysis was performed to understand the morphology of the measured signal, followed by a quantitative analysis to evaluate the influence of different parameter selection on the detection of auditory responses. Finally, both the averaging and GLM analysis techniques were used to compare the response amplitude to speech vs. noise, and for relative activation in the left vs. right cortical hemispheres. All analyses were applied to the same dataset described in Section 2.1. procedure was an averaging style analysis, and the second a finite impulse response (FIR) GLM approach. Each analysis was performed on each of the three experimental conditions. 
229 The averaging analysis consisted of several steps, starting with down-sampling the data to 3

$230 \mathrm{~Hz}$, and conversion to optical density. The scalp-coupling index (Pollonini et al., 2014) was

231 calculated for each channel between 0.7 and $1.45 \mathrm{~Hz}$, and channels with an index value below

2320.8 were removed. Data from each channel were then further cleaned by applying temporal-

233 derivative distribution repair (Fishburn et al., 2019) and short-channel regression based on

234 the nearest short channel (Saager and Berger, 2005; Scholkmann et al., 2014). Briefly, this

235 approach to short-channel regression subtracts a scaled version of the signal obtained from the nearest short channel from the signal obtained from the long channel. The modified Beer Lambert law was then applied, with a partial pathlength factor of 0.1, converting the opticaldensity measurements to changes in hemoglobin concentration. Next, channels with sourcedetector separations outside the range $20-40 \mathrm{~mm}$ were excluded, followed by application of the signal-improvement algorithm based on negative correlation between oxygenated and

241 deoxygenated hemoglobin dynamics (Cui et al., 2010). A bandpass filter was then applied 242 between 0.01 and $0.7 \mathrm{~Hz}$ with a transition bandwidth of 0.005 and $0.3 \mathrm{~Hz}$ for the low- and 243 high-pass edges, respectively. The data were cut into epochs from $3 \mathrm{~s}$ before stimulus onset 244 to $14 \mathrm{~s}$ after, and a linear detrend was applied to each epoch. Epochs with a peak-to-peak difference in any channel exceeding $100 \mu \mathrm{M}$ were then excluded. The average response per participant for each channel and for each condition was exported. 
251 In a second, and independent, analysis, data was entered into a GLM analysis using a deconvolution FIR model. This method makes no assumptions as to the shape of the hemodynamic response. Instead, a series of impulses following the onset of the stimulus are used as regressors to model the neural response. The morphology of the response can then be estimated by summing all the FIR components after multiplication by each component's weight as estimated by the GLM. See Huppert (2016) and Santosa et al. (2018) for a summary of FIR and canonical approaches within the fNIRS context.

Prior to the GLM analysis, data were down-sampled to $1 \mathrm{~Hz}$, and then converted to optical density. A lower sample rate was employed as the scalp-coupling index was not computed, and therefore, higher frequencies were not required. Next, channels with a source-detector separation outside the range $20-40 \mathrm{~mm}$ were excluded, and the modified Beer-Lambert law applied to the data, as for the averaging analysis. A GLM was then applied using a FIR model with 14 components (i.e., 14 s); this number of components was selected to ensure parity with the epoching-window approach employed in the averaging analysis. Channels were then combined into a ROI by averaging the estimates with an inverse weighting by the standard error of the GLM fit. The individual-level FIR results were then entered into a linear mixedeffects (LME) model to extract the effect of FIR delay, condition, and chromophore, whilst accounting for the random effect of subject. Santosa et al. (2018) provides for a description of these second-level statistical models. 
272

273 Next, the effect of several analysis parameters on the detection rate for auditory responses

274 was investigated. In contrast to the FIR approach (Section 2.2.1), this analysis used a 275 predefined canonical model of the evoked hemodynamic response function (HRF), specifically

276 the canonical SPM HRF, which is generated from a linear combination of two Gamma

277 functions (Penny et al., 2011). The effect of sampling rate, correction for systemic responses, and boxcar duration on the true and false-positive detection rates was explored. For simplicity, we visualized only the data for oxyhemoglobin, and not deoxyhemoglobin, signals, as the effects of different parameters was similar for both.

Only responses from optodes placed over the superior temporal gyrus were analyzed. A false positive was defined as a response detected in the (control) condition of silence. A true positive was defined as a response detected to the speech and noise conditions. Using these definitions, a receiver operating characteristic $(\mathrm{ROC})$ was defined for each analysis procedure, and the area under the curve was extracted to quantify the analysis performance. We also extracted the true positive rate (TPR) resulting from a false-positive rate (FPR) of $5 \%$, as commonly employed in clinical studies.

Specific analysis parameters were varied in this section, but each analysis consisted of the same general procedure-a re-sampling the data, followed by conversion to optical density and hemoglobin concentration. Next, channels with source-detector separation outside the 
design matrix, including the use of a $4^{\text {th }}$-order auto-regressive noise model, generating channel-level data that were used to construct a ROC curve. Channel-level data were then combined into a ROI using a weighted-average procedure, in which each channel was weighted by the inverse of the standard error of the GLM. This procedure was termed the “No Correction" analysis.

To analyze the effect of different choices of processing, several modifications were made to the procedure outlined above. Different short-channel approaches were applied to correct for systemic response, including adding the mean of the short channels as a regressor to the GLM, adding the individual short channels as regressors to the GLM, as well as adding the principal components of the short channels as regressors to the GLM (adding either a subset, or all components, were investigated). These procedures were termed the "Systemic Corrected" analysis. Similarly, the effect of sample rate was investigated by down-sampling the raw signal using different rates.

310 Finally, a group-level analysis was performed to determine if the averaging and GLM analyses both provided the same conclusion to two research questions. First, is there a difference in response amplitude between the speech and noise stimuli? And second, is there a hemispheric difference in the response to speech stimuli? We focus on group-level analysis

314 as this has been demonstrated to be reliable in auditory experiments (Wiggins et al., 2016a).

315 We also investigate whether including the approach to correcting for the systemic response correction deemed most effective (see Section 2.3) modifies experimental conclusions. 
For the averaging analysis, the same approach was made as in Section 2.2, after which, the

321 mean value between 5 and $7 \mathrm{~s}$ of the average waveform for each participant was exported for analysis by statistical testing.

\subsubsection{Canonical model analysis}

325

326 For the canonical-model GLM analysis, two procedures were used; the No Correction approach and the Systemic Corrected approach, the latter of which included all principal components as regressors in the GLM to compensate for systemic responses. Both analyses used a sample rate of $0.6 \mathrm{~Hz}$ and a $3 \mathrm{~s}$ duration for the boxcar function.

\subsubsection{Statistical analysis}

332

333 To summarize the dataset, results from the Systemic Corrected approach were entered into

334 a linear mixed-effects model that accounted for condition, ROI, and chromophore with 335 participant as a random variable. In Roger-Wilkinson notation this would be described as $\beta$ $336-1+$ Condition:ROI:Chroma + (1|ID).

337 For each of the three analyses described above (averaging, GLM No Correction, GLM Systemic 338 Corrected), a response estimate was exported for each participant, each condition, and each 
ROI. These data were then used to address two issues. First, using all channels over both left and right superior temporal gyri as a single ROI, a linear mixed-effects model was used to determine if the response to speech was different from that to noise. Participant was included as a random effect. In Roger-Wilkinson notation this is described as $\beta \sim$ Condition + (1|ID).

343 Second, a linear mixed-effects model was used to determine if the left superior temporal 344 gyrus shows a different response amplitude to the right in the speech condition, described as

$345 \beta \sim \mathrm{ROI}+(1 \mid \mathrm{ID})$ in Roger-Wilkinson notation.

\section{Results \& Discussion}

347 To ensure that the filter was parameterized correctly, as to remove unwanted components

348 of the measurements and retain the frequency content of interest, the spectrum of the raw

349 fNIRS data extracted from an example data file is plotted along with the expected

350 hemodynamic response (Figure 2). The spectral content of the model boxcar function of the

351 experiment convolved with a model neural response (Figure 2, red curve) indicates that the majority of the signal content is around $0.05 \mathrm{~Hz}$, consistent with the average presentation rate of the experiment. The spectral content of an example measurement (Figure 2, black curve) indicates a clear signal generated by the systemic pulse rate of around $1 \mathrm{~Hz}$. The filterfrequency response (Figure 2, blue) clearly retains the peak of the expected response, but excludes the low-frequency drift and high-frequency (pulse-rate) components. 


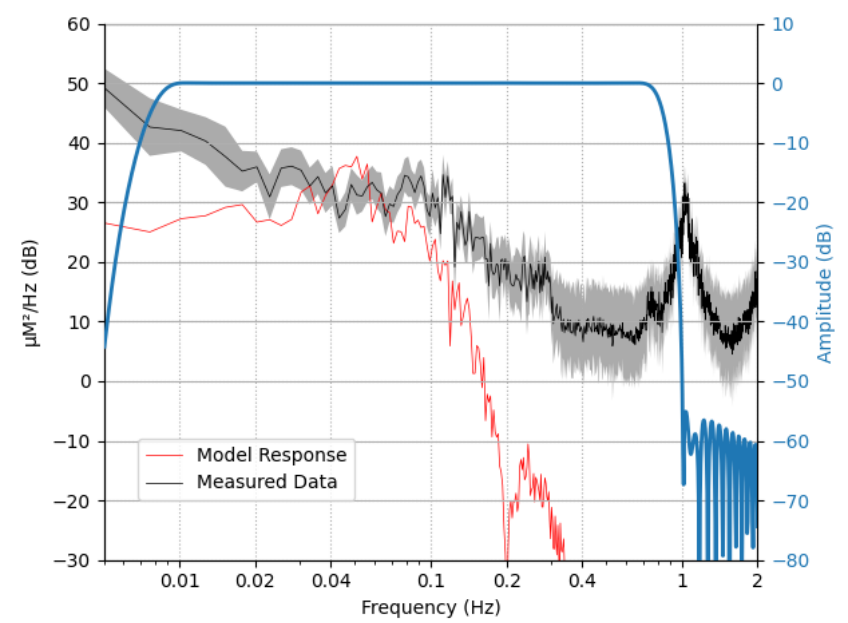

Figure 2: Summary of frequency information. The frequency content of the expected neural response based on trigger information and model hemodynamic response function is shown in red (arbitrary scaling). The applied filter is shown in blue. Raw data from an example file is shown in black, with the solid line indicating the mean value across all channels and the shading representing $95 \%$ confidence intervals across channels. Note that the filter retains most of the experimental frequency content while removing high-frequency heart rate content (around $1 \mathrm{~Hz}$ ) and low frequency content in the measured data.

Two approaches were applied to investigate the morphology of responses to auditory stimuli in each ROI. Here, we provide a qualitative description of morphology.

372 To summarize the group-level averaging analysis results, a time series visualizes the average

373 signal across participants and a bootstrapped $95 \%$ confidence band around the mean for each

374 condition and ROI (Figure 3). Responses were observed in the STG regions for both noise and speech stimuli, but not for the silent conditions. For the silence condition, flat measurements were observed over the entire waveform in all ROls. For both speech and noise conditions,

377 the largest responses were measured from optodes placed over the left and right superior 
temporal gyri. These responses show a canonical hemodynamic response, with a peak

379 response around 5- to 7-s after stimulus onset, consistent with the duration of the stimulus.

380 As such, only channels over the superior temporal gyri were used subsequently to quantify response morphology.
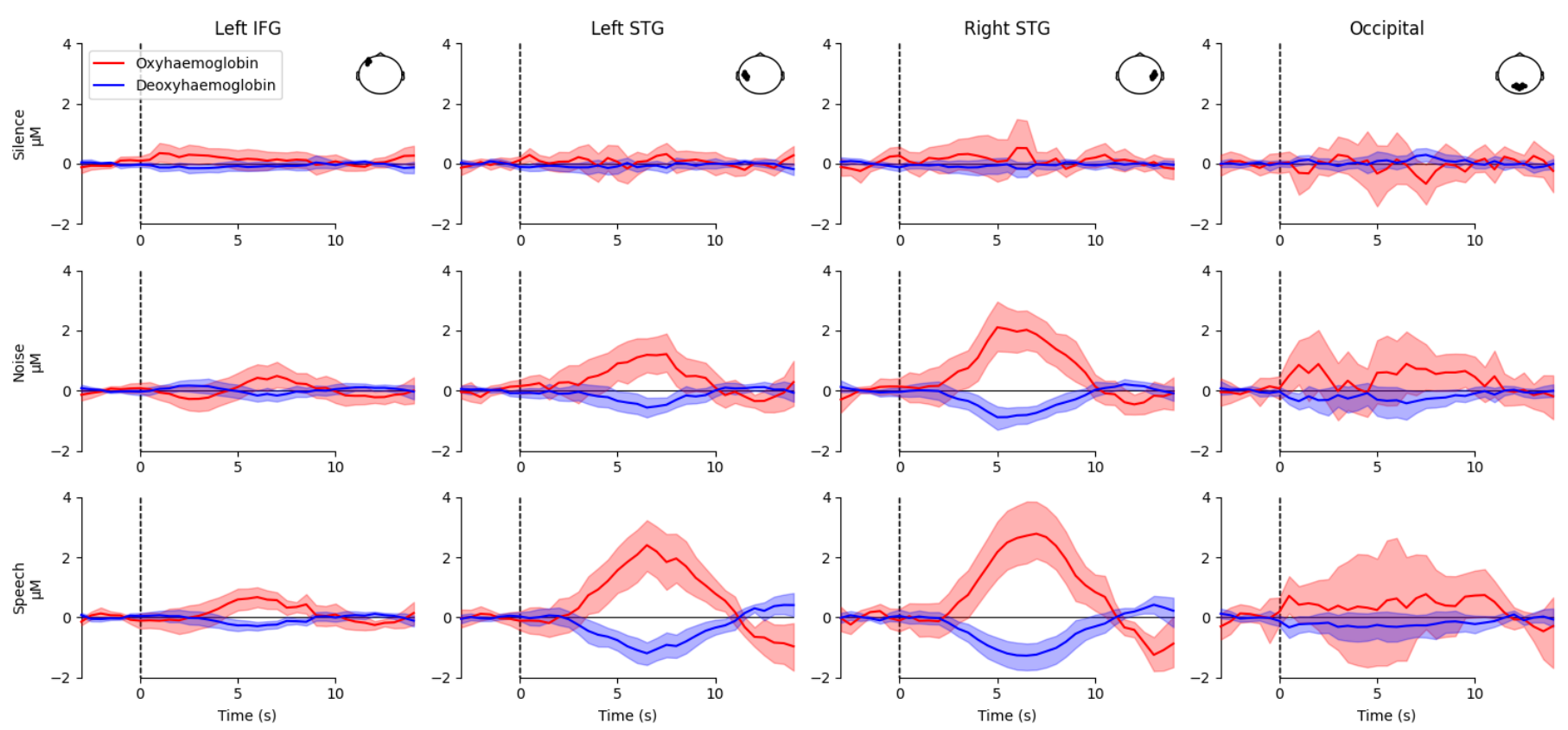

Figure 3: Morphology of auditory fNIRS responses using the averaging approach for all regions of interest and conditions.

Each column represents a different region of interest as illustrated in the top down head view inset. Each row represents a different stimulus condition. Red represents oxyhemoglobin, blue represents deoxyhemoglobin. Shaded lines indicate 95\% confidence intervals. Responses were observed over the left and right superior temporal gyrus (STG) for both speech and noise conditions, but not for silence.

389

392 A FIR GLM analysis was also used to examine the morphology of the hemodynamic response,

393 using only optodes situated over the superior temporal gyri. A comparison of the estimated 

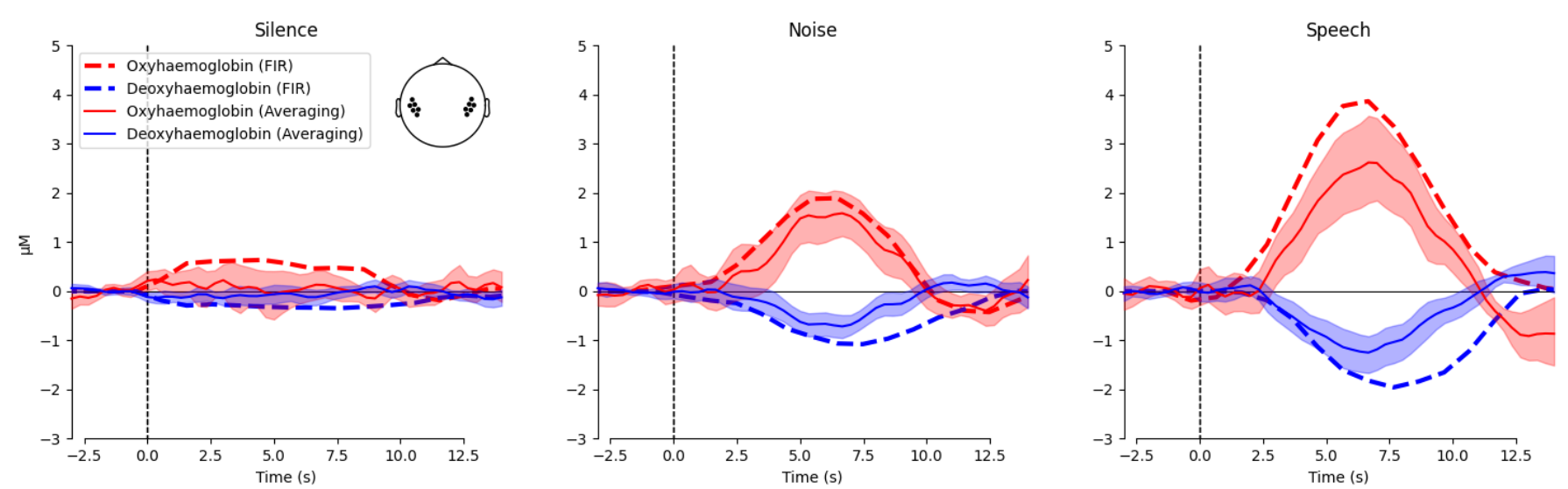

Figure 4: Morphology of auditory fNIRS responses over the superior temporal gyrus. Each column represents a different stimulus condition. Responses are illustrated for both oxy-and deoxyhemoglobin, red and blue respectively. The shaded areas and solid line represent the mean and 95\% confidence intervals for the averaging approach. The dashed lines illustrate the estimates for the FIR GLM approach. Note that the averaging and FIR GLM fits are quite similar, except for a larger estimate

405

407 We next examined the effect of different analysis parameters on the detection of responses in individual participants. ROC curves for both ROIs (Figure 5a) and individual channels (Figure 5b) indicates ROls show greater sensitivity to true positives than individual channels, likely due to noisy channels being inversely weighted. Subsequently, we focus on the channel-level results (Figure 5c). under the curve (AUC) measure. A larger value indicates better performance across the entire 
metric, because this tends to be more relevant for clinical purposes. Many of the differences in the ROC occur at a high FPR at and above 50\%, however, this FPR would be considered unacceptable in a clinical setting.

\subsubsection{Effect of short channel regression on detection of auditory responses}

421

We first examined the effect of different short-channel based methods of reducing systemic responses from the estimated neural responses. The effect of adding different representations of the short channels as regressors in the GLM is explored. These representations include a limited number of principal components, all principal components, the individual short channels, or the mean of the short channels per each chromophore.

427 Without short-channel correction, responses were detected in less than $20 \%$ of measurements for a false-positive rate of $5 \%$. As expected, applying the short-channel method to remove systemic components resulted in a substantial improvement to the detection rate (Santosa et al., 2020; Scholkmann et al., 2014; Tak and Ye, 2014; Wyser et al., 2020). Although it is common to use just the first or second principal components as regressors (Weder et al., 2020), we observed that including all components resulted in the best performance, consistent with Santosa et al. (2020).

434 We also observed that including all the short channels or the mean as regressors, instead of the principal components, also results in good detection rates. Whilst we observed no effect of including all principal components or just individual channels, we selected the principal components for subsequent analysis, as this is suggested to be the most effective method to compensate for systemic components in the estimation of neural responses (Santosa et al., 
bioRxiv preprint doi: https://doi.org/10.1101/2020.12.22.423886; this version posted December 22, 2020 . The copyright holder for this

preprint (which was not certified by peer review) is the author/funder. All rights reserved. No reuse allowed without permission.

to implement, describe, and replicate.

441

(a)

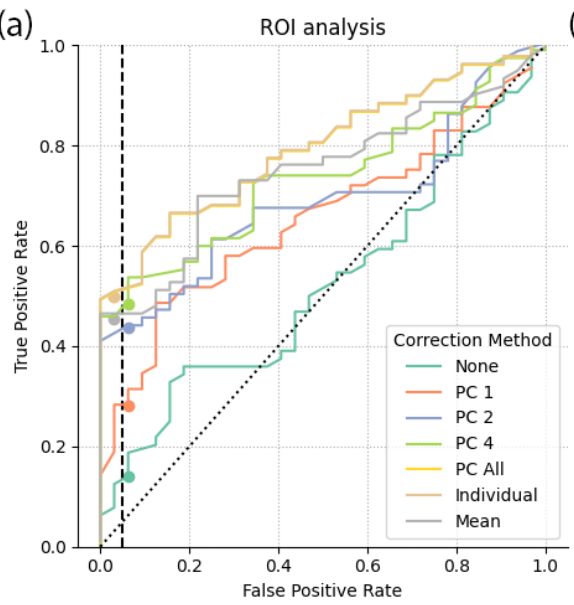

(b)

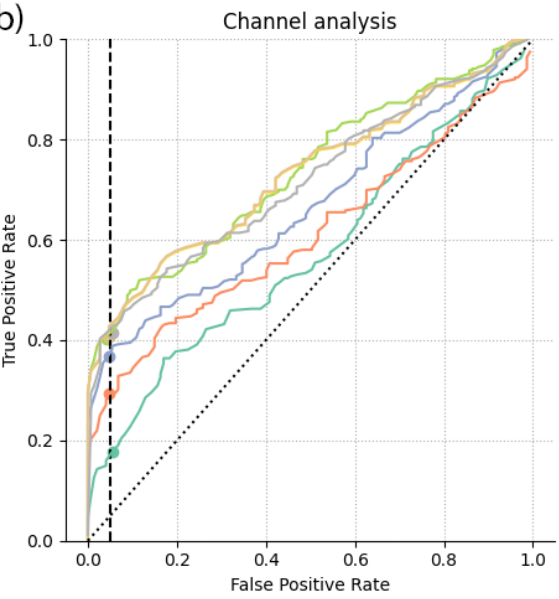

(c)

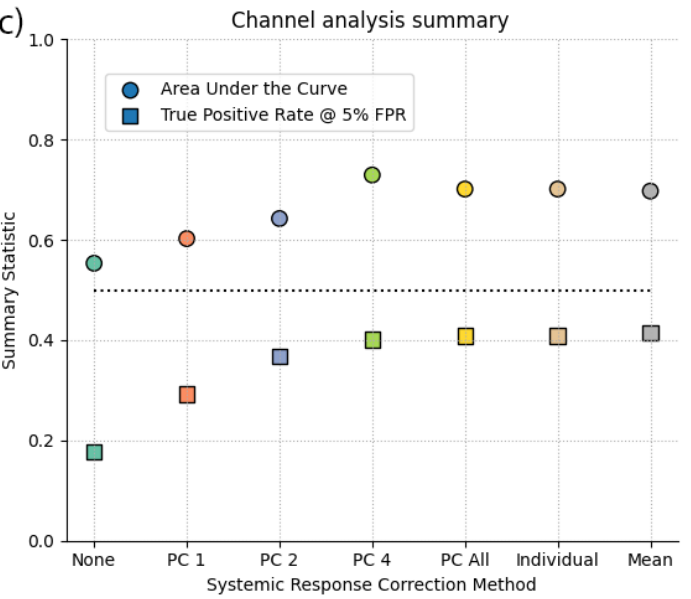

443

444

445

446

447

448

449

450

451

452

453

454

455

456

457

458

Figure 5: The effect of systemic response correction on auditory fNIRS response estimates. Receiver operating characteristic curves for the superior temporal gyri region of interest (a) and individual channels over the superior temporal gyri (b). Summary statistics from the individual channel ROC (c) with area under the curve (circle) and true positive rate at $5 \%$ false positive rate (square) metrics for each method. Analysis with no systemic correction is included as a reference (green), analysis with 1, 2, 4, or all principal components $(P C)$ of the short channels as regressors in the GLM is shown (orange, blue, light green, yellow), all short channels included as individual regressors (brown) or averaged per chromophore (gray). Note that all systemic response correction approaches provide improved detection over no correction. Including all principal components, the mean of the short channels, or all individual channels provides best auditory response detection.

\subsubsection{Effect of sample rate on the detection of auditory responses}

fNIRS devices often require a trade-off between the number of channels and acquisition sample-rate, and understanding the effect of this trade-off is of practical concern for auditory experiments; performance generally decreases with lower sample rates (Figure 6). Analysis of data with a higher sample rate requires more memory and computational resources, so we selected $0.6 \mathrm{~Hz}$ as a sample rate that balances computational cost with accuracy. 

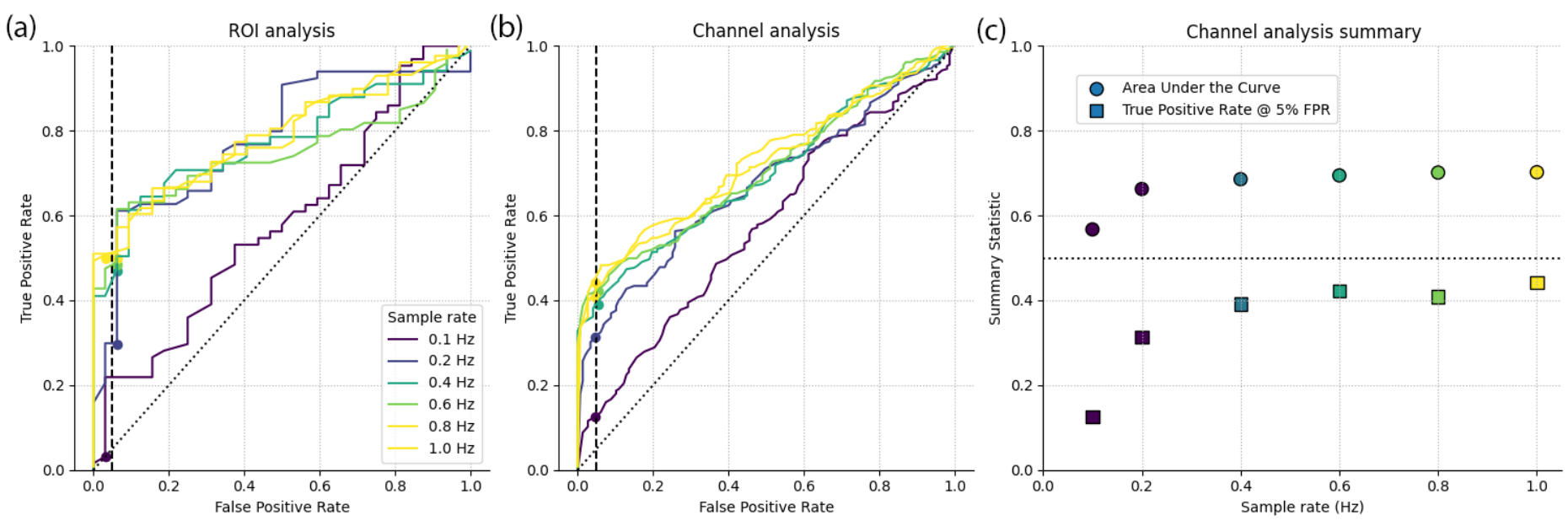

461

462

463

464

465

466

467

468

469

470

471

472

473

474

475

476

477

478

479

Figure 6: The effect of sample rate on auditory fNIRS response estimates. Receiver operating characteristic curves for the superior temporal gyri region of interest (a) and individual channels over the superior temporal gyri (b). Summary statistics from the individual channel ROC (c) with area under the curve (circle) and true positive rate at $5 \%$ false positive rate (square) metrics for data sampled at different rates. Analysis indicates improved performance with increasing sample rate, but with limited improvement above approximately $0.6 \mathrm{~Hz}$.

\subsubsection{Effect of boxcar duration on the detection of auditory responses}

The fNIRS responses to our 5-s block stimuli peaks around 6 to $7 \mathrm{~s}$ after stimulus onset (Figure

4). GLM analyses fit an expected neural response to the data, in which the expected neural response is generated by convolving a model HRF with a boxcar function generated from the onset times of the stimuli. The length of the boxcar function can be varied to account for the duration of the neural response, and is typically set to the duration of the stimulus. However, response morphology can change with stimuli and brain location. As such, we investigated the effect of boxcar length on response detection to auditory stimuli, and find that the 3-s boxcar function provides the greatest true positive rate, for a pre-determined $5 \%$ falsepositive rate (Figure 7). Note, however, that the reduction in performance that comes from using swapping out 3-s boxcar function for one of 1-s or 5-s duration is smaller than the reduction in performance that comes about by not employing systemic correction, or when 
too low a sample rate is used. An alternative approach to account for differences between

481 the model and the measured response is to include a derivative term in the design matrix

482 (Mushtaq et al., 2020; Zhang et al., 2020). However, since we observed good correspondence

483 between the response morphology and the expected canonical response, we did not include

484 derivative terms in our analysis.

485
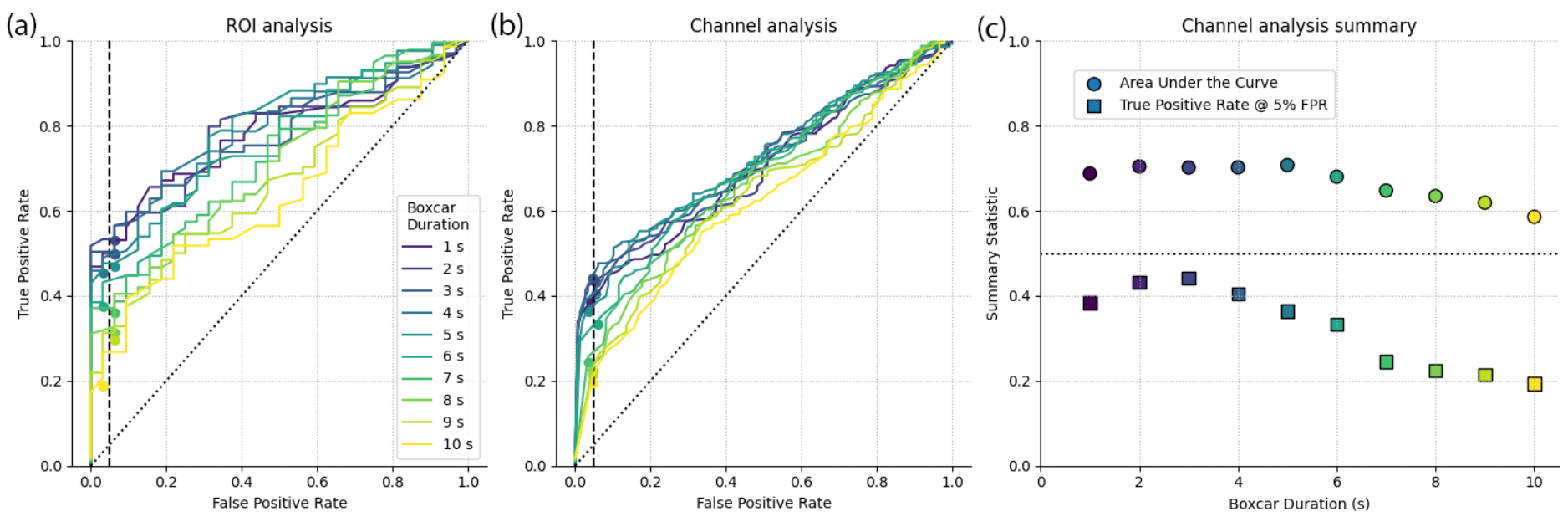

Figure 7: The effect of boxcar function duration on auditory fNIRS response estimates. Receiver operating characteristic curves

for the superior temporal gyrus region of interest (a) and individual channels over the superior temporal gyrus (b). Summary statistics from the individual channel ROC (c) with area under the curve (circle) and true positive rate at $5 \%$ false positive rate

490 (square) metrics for different boxcar durations. Analysis indicates optimal detection rates for a $3 \mathrm{~s}$ boxcar function, note that

491

the stimulus duration was $5 \mathrm{~s}$.

492

Additional analysis parameters beyond the scope of the current study include effects arising

494 from selection of the specific auto-regressive model (Huppert (2016), or alternate canonical

495 functions (Glover (1999). Based on the data thus far, we maintained a sample rate of $0.6 \mathrm{~Hz}$

496 in future analyses, and included all principal components as regressors, employing a 3-s

497

boxcar function to model the hemodynamic response. 
500 Finally, we investigated whether, when applied at a group level, the averaging and GLM

501 approaches to fNIRS analysis provide for the same experimental conclusions. Two common

502 questions in auditory experiments were explored. First, could we detect a difference in response amplitude between two conditions, in this example: speech and noise. And second,

504 within one condition, is a difference in response amplitudes apparent across brain hemispheres, often termed "lateralization of responses."

We first summarized the dataset (GLM analogue of Figure 2) by modelling the response

507 amplitude as a factor of ROI, condition, and chromophore in a LME model, with participant

509 significant responses were obserbed in either the left inferior frontal gyrus or occipital cortice,

510 and the silent, control condition generated no responses in any ROI. Significant responses

511 were observed to both speech and noise in the two ROIs of superior temporal gyrus. The lack

512 of any detectable response to speech stimuli in left inferior frontal gyrus may be due to the

513 passive nature of the experimental task; this cortical region has been indicated in the processing of speech, particularly in active tasks with more challenging acoustic conditions. 

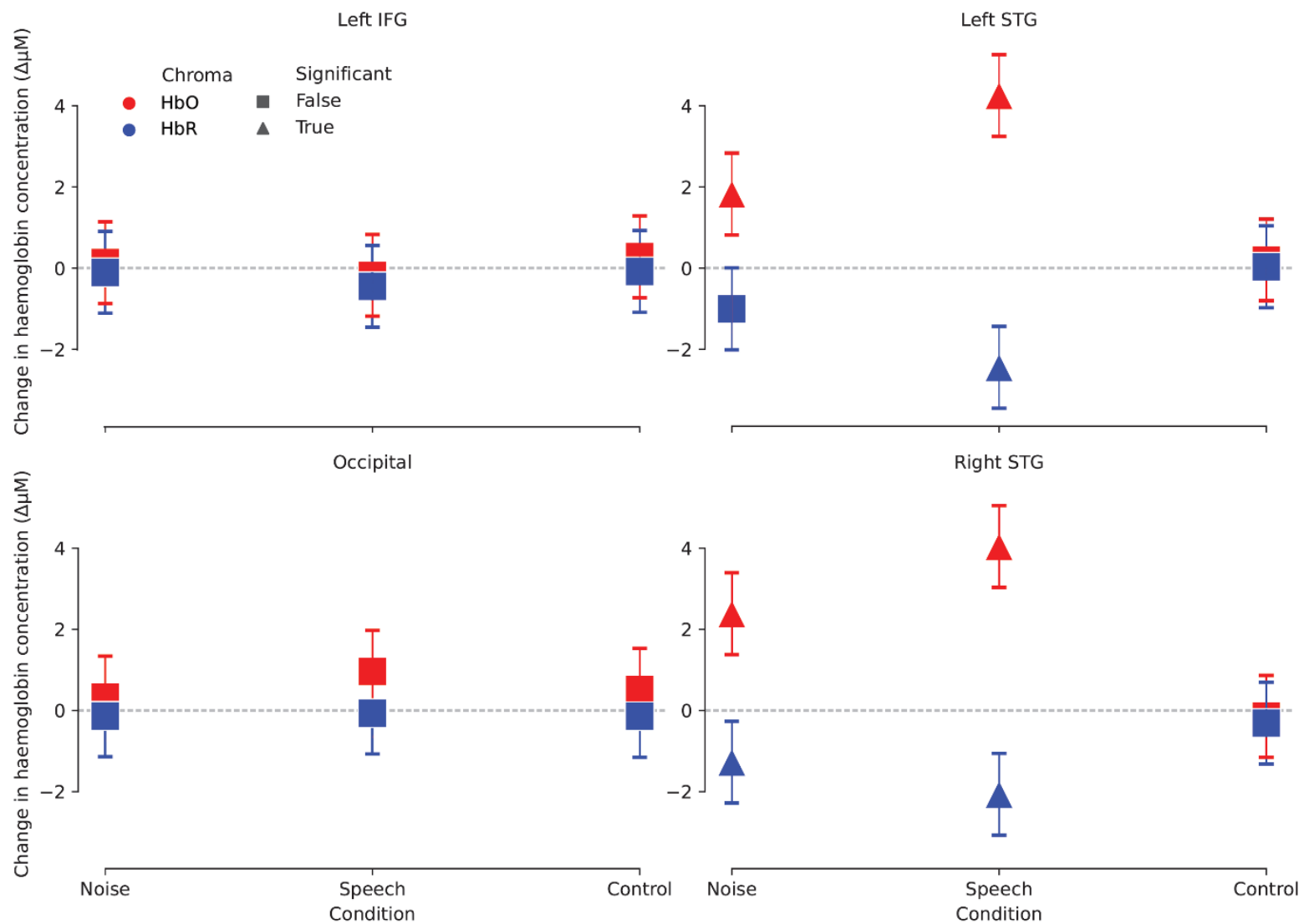

517 Figure 8: Estimates of response per condition and region of interest using the GLM analysis. Oxy-and deoxyhemoglobin 518 responses are shown in red and blue respectively. The presence of a response (statistical difference to zero) is indicated by a 519 triangle. Error bars represent the 95\% confidence intervals of the mean.

523 We next addressed the questions of whether responses to speech are larger than responses activation are observed. 

response was $2.043 \mu \mathrm{M}$ larger than that evoked by noise $(p<.001)$. Using the average waveform amplitude 5 to $7 \mathrm{~s}$ post stimulus onset, we observed that the estimated response to speech was $1.0 \mu \mathrm{M}$ larger than to the noise $(p<.01)$. From this, we conclude that both analysis methods generate the same experimental conclusion, consistent with visual inspection of the averaging and FIR GLM analyses (Figure 3). The estimated response amplitude difference was larger for the GLM approach, possibly due to this approach better accounting for the statistical nature of the fNIRS noise (Huppert, 2016). The time window used in the averaging approach may also reduce the estimated response amplitude, whereas a peak picking approach may result in a slightly larger estimate of the response. However, automated peak-picking approaches are prone to error, particularly when the signal-to-noise ratio is low, whilst manual methods of peak-picking reduce the repeatability of an analysis.

\subsubsection{Effect of systemic component rejection}

545 Analyzing the data using the GLM approach, with no correction for systemic responses-the No Correction analysis-indicates that the speech response was $2.306 \mu \mathrm{M}$ larger than that to

547 the noise stimulus $(p=.025)$. Not including corrections for systemic responses generated a 548 similar effect size to the Systemic Corrected analysis. This correspondence between methods 549 of analysis may be due to the systemic response being relatively small, or the systemic response being similar across conditions. Our experiment was a passive listening-task, and 
event-locked systemic component to auditory stimuli required participants to generate a response, for example, by means of a button press (Shoushtarian et al., 2019). These, moreactive, experimental paradigms may generate a larger systemic component, and therefore elicit greater differences between analyses corrected or uncorrected for systemic effects.

561 Finally, to address whether a difference in response amplitude exists between left and right in a LME model examining the effect of ROI, with participant as a random effect. The model reported that estimated amplitude of the fNIRS response in the right hemisphere was not significantly different to that in the left $(\beta=-0.21, p=.73)$. Similarly, the same LME model reported no significant lateralization of the response amplitude when the averaging analysis was employed $(\beta=1.0, p=.13)$.

571 When assessing the No Correction GLM data at a group level, no significant effect of

572 lateralization was observed $(\beta=0.18, p=.87)$, indicating that not compensating for systemic

573 components does not generate aberrant lateralization effects. However, we cannot conclude 
574 from these data that, if a lateralization effect were present, it would be detectable without 575 systemic correction.

576

577 4. Conclusion

578 A reference block-design auditory fNIRS dataset was created with two common acoustic 579 stimuli. Using this dataset, it was determined that both an averaging approach and a FIR GLM 580 analysis resulted in similar response morphology. The effect of correcting for systemic 581 hemodynamic responses using short optical channels was evaluated on the response 582 detection of the GLM approach, where it was determined that including the individual short 583 channels, or the principal components of the short channels, resulted in similar practical

584 improvements to detection. At a group level, it was observed that both the averaging and 585 GLM approach produced the same experimental conclusions to two common research questions. Not including short-channel corrections did not change the group-level conclusions. This may be due to the fact that the task was passive in nature, and may not hold for experiments requiring active participation.

The fNIRS data reported in this article will be released on OSF.io and github.com in the BIDS data format to allow ease of reuse (Gorgolewski et al., 2016). All the code functions used in this analysis are available at mne.tool/mne-nirs and the associated GitHub page, along with example analysis tutorials. 


\section{Acknowledgments/Funding Sources}

597 This study was supported by an Australian Research Council Laureate Fellowship (No.

598 FL160100108) awarded to David McAlpine. E. Larson was supported by the National Institutes

of Health under Grant R01NS104585-01A1.

600

601

\section{Bibliography}

602

603 Abraham, A., Pedregosa, F., Eickenberg, M., Gervais, P., Mueller, A., Kossaifi, J., Gramfort, A., 604 Thirion, B., Varoquaux, G., 2014. Machine learning for neuroimaging with scikit-learn. Front 605 Neuroinform 8, 14.

606 Anderson, C.A., Wiggins, I.M., Kitterick, P.T., Hartley, D.E., 2017. Adaptive benefit of cross607 modal plasticity following cochlear implantation in deaf adults. Proceedings of the National 608 Academy of Sciences 114, 10256-10261.

609 Anderson, C.A., Wiggins, I.M., Kitterick, P.T., Hartley, D.E., 2019. Pre-operative brain imaging 610 using functional near-infrared spectroscopy helps predict cochlear implant outcome in deaf 611 adults. Journal of the Association for Research in Otolaryngology 20, 511-528.

612 Barker, J.W., Aarabi, A., Huppert, T.J., 2013. Autoregressive model based algorithm for 613 correcting motion and serially correlated errors in fNIRS. Biomed Opt Express 4, 1366-1379.

614 Basura, G.J., Hu, X.S., Juan, J.S., Tessier, A.M., Kovelman, I., 2018. Human central auditory 615 plasticity: A review of functional near-infrared spectroscopy (fNIRS) to measure cochlear 616 implant performance and tinnitus perception. Laryngoscope investigative otolaryngology 3 , 617 463-472.

618 Brockway, J.P., 2000. Two functional magnetic resonance imaging $f(M R I)$ tasks that may 619 replace the gold standard, Wada testing, for language lateralization while giving additional 620 localization information. Brain Cogn 43, 57-59. 
621 Cohen, M.S., 1997. Parametric analysis of fMRI data using linear systems methods. 622 Neuroimage 6, 93-103.

623 Cui, X., Bray, S., Bryant, D.M., Glover, G.H., Reiss, A.L., 2011. A quantitative comparison of

624 NIRS and fMRI across multiple cognitive tasks. Neuroimage 54, 2808-2821.

625 Cui, X., Bray, S., Reiss, A.L., 2010. Functional near infrared spectroscopy (NIRS) signal 626 improvement based on negative correlation between oxygenated and deoxygenated 627 hemoglobin dynamics. Neuroimage 49, 3039-3046.

628 Dale, A.M., Buckner, R.L., 1997. Selective averaging of rapidly presented individual trials using 629 fMRI. Hum. Brain Mapping, 329-340.

630 Dawson, G.D., 1954. A summation technique for the detection of small evoked potentials. 631 Electroencephalography and Clinical Neurophysiology 6, 65-84.

632 Dawson, P.W., Hersbach, A.A., Swanson3, B.A., 2013. An Adaptive Australian Sentence Test 633 in Noise (AuSTIN). Ear and Hearing 34, 592-600.

634 Fabbri, F., Sassaroli, A., Henry, M.E., Fantini, S., 2004. Optical measurements of absorption 635 changes in two-layered diffusive media. Phys Med Biol 49, 1183-1201.

636 Fishburn, F.A., Ludlum, R.S., Vaidya, C.J., Medvedev, A.V., 2019. Temporal Derivative 637 Distribution Repair (TDDR): A motion correction method for fNIRS. Neuroimage 184, 171-179.

638 Gervain, J., Macagno, F., Cogoi, S., Pena, M., Mehler, J., 2008. The neonate brain detects 639 speech structure. Proc Natl Acad Sci U S A 105, 14222-14227.

640 Glover, G.H., 1999. Deconvolution of impulse response in event-related BOLD fMRI. 641 Neuroimage 9, 416-429.

642 Gorgolewski, K.J., Auer, T., Calhoun, V.D., Craddock, R.C., Das, S., Duff, E.P., Flandin, G., Ghosh, 643 S.S., Glatard, T., Halchenko, Y.O., 2016. The brain imaging data structure, a format for 644 organizing and describing outputs of neuroimaging experiments. Scientific data 3, 1-9.

645 Gramfort, A., Luessi, M., Larson, E., Engemann, D.A., Strohmeier, D., Brodbeck, C., Goj, R., Jas, 646 M., Brooks, T., Parkkonen, L., Hamalainen, M., 2013. MEG and EEG data analysis with MNE647 Python. Front Neurosci 7, 267. 

L., Hamalainen, M.S., 2014. MNE software for processing MEG and EEG data. Neuroimage 86, 446-460.

651

652

Huppert, T.J., 2016. Commentary on the statistical properties of noise and its implication on general linear models in functional near-infrared spectroscopy. Neurophotonics 3, 010401.

Kriegeskorte, N., Simmons, W.K., Bellgowan, P.S., Baker, C.I., 2009. Circular analysis in systems neuroscience: the dangers of double dipping. Nat Neurosci 12, 535-540.

Lawrence, R.J., Wiggins, I.M., Anderson, C.A., Davies-Thompson, J., Hartley, D.E.H., 2018. Cortical correlates of speech intelligibility measured using functional near-infrared spectroscopy (fNIRS). Hear Res 370, 53-64.

Mushtaq, F., Wiggins, I.M., Kitterick, P.T., Anderson, C.A., Hartley, D.E.H., 2019. Evaluating time-reversed speech and signal-correlated noise as auditory baselines for isolating speechspecific processing using fNIRS. PLoS One 14, e0219927.

Mushtaq, F., Wiggins, I.M., Kitterick, P.T., Anderson, C.A., Hartley, D.E.J.F.i.H.N., 2020. The Benefit of Cross-Modal Reorganization on Speech Perception in Pediatric Cochlear Implant Recipients Revealed Using Functional Near-Infrared Spectroscopy. 14.

Penny, W.D., Friston, K.J., Ashburner, J.T., Kiebel, S.J., Nichols, T.E., 2011. Statistical parametric mapping: the analysis of functional brain images. Elsevier.

Pollonini, L., Olds, C., Abaya, H., Bortfeld, H., Beauchamp, M.S., Oghalai, J.S., 2014. Auditory cortex activation to natural speech and simulated cochlear implant speech measured with functional near-infrared spectroscopy. Hear Res 309, 84-93.

Rombouts, S.A., Barkhof, F., Hoogenraad, F.G., Sprenger, M., Valk, J., Scheltens, P., 1997. Testretest analysis with functional MR of the activated area in the human visual cortex. AJNR Am J Neuroradiol 18, 1317-1322.

Rovetti, J., Goy, H., Pichora-Fuller, M.K., Russo, F.A., 2019. Functional Near-Infrared Spectroscopy as a Measure of Listening Effort in Older Adults Who Use Hearing Aids. Trends Hear 23, 2331216519886722. Underlying Brain Activity? Trends Hear 22, 2331216518804116. 
678 Saager, R.B., Berger, A.J., 2005. Direct characterization and removal of interfering absorption 679 trends in two-layer turbid media. J Opt Soc Am 22, 1874-1882.

680 Santosa, H., Fishburn, F., Zhai, X., Huppert, T.J., 2019. Investigation of the sensitivity681 specificity of canonical- and deconvolution-based linear models in evoked functional near682 infrared spectroscopy. Neurophotonics 6, 025009.

683 Santosa, H., Zhai, X., Fishburn, F., Huppert, T., 2018. The NIRS Brain AnalyzlR Toolbox. 684 Algorithms 11. correction techniques for removing systemic physiological signal in functional near-infrared spectroscopy studies. Neurophotonics 7, 035009.

Scholkmann, F., Metz, A.J., Wolf, M., 2014. Measuring tissue hemodynamics and oxygenation by continuous-wave functional near-infrared spectroscopy--how robust are the different calculation methods against movement artifacts? Physiol Meas 35, 717-734. Neuroimaging with near-infrared spectroscopy demonstrates speech-evoked activity in the auditory cortex of deaf children following cochlear implantation. Hear Res 270, 39-47.

694 Shoushtarian, M., Alizadehsani, R., Khosravi, A., Acevedo, N., McKay, C.M., Nahavandi, S., 695 Fallon, J.B., 2020. Objective measurement of tinnitus using functional near-infrared 696 spectroscopy and machine learning. PLoS One 15, e0241695.

697 Shoushtarian, M., Weder, S., Innes-Brown, H., McKay, C.M., 2019. Assessing hearing by 698 measuring heartbeat: The effect of sound level. PLoS One 14, e0212940.

699 Stoppelman, N., Harpaz, T., Ben-Shachar, M., 2013. Do not throw out the baby with the bath 700 water: choosing an effective baseline for a functional localizer of speech processing. Brain 701 Behav 3, 211-222.

702 Tachtsidis, I., Scholkmann, F., 2016. False positives and false negatives in functional near703 infrared spectroscopy: issues, challenges, and the way forward. Neurophotonics 3, 031405. 

fNIRS Responses Can Be Better Explained by Loudness Percept than Sound Intensity. Ear Hear 41, 1187-1195.

709

710

711

712

713

714

715

716

717

718

719

720

721

722

723

724

725

726

727

728

729

730

Zimeo Morais, G.A., Balardin, J.B., Sato, J.R., 2018. fNIRS Optodes' Location Decider (fOLD): a toolbox for probe arrangement guided by brain regions-of-interest. Sci Rep 8, 3341.

Weder, S., Zhou, X., Shoushtarian, M., Innes-Brown, H., McKay, C., 2018. Cortical Processing Related to Intensity of a Modulated Noise Stimulus-a Functional Near-Infrared Study. J Assoc Res Otolaryngol 19, 273-286.

Wiggins, I.M., Anderson, C.A., Kitterick, P.T., Hartley, D.E., 2016a. Speech-evoked activation in adult temporal cortex measured using functional near-infrared spectroscopy (fNIRS): Are the measurements reliable? Hear Res 339, 142-154.

Wiggins, I.M., Hartley, D.E., 2015. A synchrony-dependent influence of sounds on activity in visual cortex measured using functional near-infrared spectroscopy (fNIRS). PLoS One 10, e0122862.

Wiggins, I.M., Wijayasiri, P., Hartley, D., 2016b. Shining a light on the neural signature of effortful listening. The Journal of the Acoustical Society of America 139, 2074-2074.

Wijayasiri, P., Hartley, D.E.H., Wiggins, I.M., 2017. Brain activity underlying the recovery of meaning from degraded speech: A functional near-infrared spectroscopy (fNIRS) study. Hear Res 351, 55-67.

Wyser, D., Mattille, M., Wolf, M., Lambercy, O., Scholkmann, F., Gassert, R., 2020. Shortchannel regression in functional near-infrared spectroscopy is more effective when considering heterogeneous scalp hemodynamics. Neurophotonics 7, 035011.

Yücel, M.A., Selb, J.J., Huppert, T.J., Franceschini, M.A., Boas, D.A.J.C.o.i.b.e., 2017. Functional near infrared spectroscopy: enabling routine functional brain imaging. 4, 78-86.

Zhang, M., Alamatsaz, N., Ihlefeld, A., 2020. Hemodynamic responses link individual differences in informational masking to the vicinity of superior temporal gyrus. 2020.2008.2021.261222.

Zhang, M., Mary Ying, Y.L., Ihlefeld, A., 2018. Spatial Release From Informational Masking: Evidence From Functional Near Infrared Spectroscopy. Trends Hear 22, 2331216518817464. 